\section{Diabetes CME}

Editor - Thank you for the excellent CME articles on diabetes (Clin Med December 2014 pp 663-82). It was helpful to read about situations where diabetes might be difficult to manage such as in pregnancy, HIV and rare genetic subtypes of diabetes. Could we highlight another group of patients not discussed in the aforementioned CME articles but who may also have complex diabetes care?

It is increasingly recognised that diabetes care is challenging in the palliative care population. Patients with life-limiting diagnoses and a short prognosis may have diabetes as a comorbidity, and difficult glycaemic control can occur due to the complications of advanced disease.

Achieving acceptable glycaemic control is difficult for a number of reasons. In addition to the altered metabolism seen in cachexia and anorexia, we encounter other concerns in our practice. For example, renal and hepatic impairment may result in hypoglycaemia due to prolongation of action of oral hypoglycaemic agents and insulin. Corticosteroids, used as part of chemotherapy regimens or to palliate symptoms such as fatigue, can cause hyperglycaemia. Finally, medically assisted nutrition via nasogastric or gastrostomy tubes (as seen in patients with motor neurone disease or head and neck cancer) requires careful titration and timing of administration of insulin.

Avoidance of symptomatic hyperglycaemia and hypoglycaemia is important in this population, particularly in the final weeks and days of life. Hyperglycaemia can result in dry mouth, thirst and agitation. Conversely, persistent hypoglycaemia is a potentially treatable cause of deterioration in an otherwise stable patient.

There is debate around what constitutes 'acceptable glycaemic control' and how frequently blood sugars should be monitored, especially in actively dying patients. A suggested monitoring regime, as well as practical advice on management related to prognostic groupings is outlined in End of life diabetes care, ${ }^{1}$ a guidance document commissioned by Diabetes UK. The guidance suggests glucose control targets of between $6-15 \mathrm{mmol} / \mathrm{l}$. However, further work still needs to be done to establish best practice in this challenging and diverse population.

CATHERINE GWILT Specialist registrar in palliative medicine, Royal Surrey County Hospital NHS Foundation Trust, Guildford, UK

OMAR MUSTAFA Consultant physician in diabetes and general medicine, Kings College Hospital NHS Foundation Trust, London, UK

EMMA HALL Consultant in palliative medicine, St Christopher's Hospice, London, UK

\section{References}

1 Diabetes UK. End of life diabetes care, clinical care recommendations, 2nd edn (2013). Available online at www.diabetes.org.uk/upload/ Position\%20statements/End-of-life-care-Clinical-recs111113.pdf [Accessed 15 January 2015].

\section{Sudden onset postural livedo reticularis, cyanotic toes} and multiorgan failure

Editor - I read with great interest the remarkable text of lesson of the month in 'Sudden onset postural livedo reticularis, cyanotic toes and multiorgan failure' (Clin Med June 2014 pp 314-5).

Although the case was typical of rare cholesterol embolisation syndrome (CES), the text in my opinion was lacking references to the following:

$>$ workup of the hypercoaguable status of the catastrophic antiphospholipid syndrome ${ }^{1}$ which can share clinical features of CES, in particular the acute development of livedo reticularis. Therefore, the relevant tests for such potential diagnosis, if any had been carried out, would have been worthwhile mentioning in the text

> hypereosinophilia, which is known to occur in up to $80 \%$ of $\mathrm{CES}^{2}$

> use of antiplatelet agents, and/or angiotensin-converting enzymes and angiotensin receptor blockers ${ }^{2}$ in the management of the case. Use would seem reasonable as these agents tend to prevent other adverse cardiovascular events such as myocardial infarction, a leading cause of death in patients with atherosclerosis.,

Despite all the above I still commend the authors who elegantly presented a rare but very serious condition.

HAIDER M AL-ATTIA

Consultant physician in internal medicine and rheumatology, Universal Hospital, Abu Dhabi, UAE

\section{References}

1 Aguiar CL, Erkan D. Catastrophic antiphospholipid syndrome: how to diagnose a rare but highly fatal disease. Ther Adv Musculoskelet Dis 2013;5:305-14.

2 Kronson I, Saric M. Cholesterol Embolization Syndrome. Circulation 2010;122:631-41.

3 The Heart Outcomes Prevention Evaluation Study Investigators. Effects of an angiotensin-converting enzyme inhibitor, ramipril, on cardiovascular events in high-risk patients. N Eng J Med 2000;342:145-53.

4 Belenfant X, Meyrier A, Jacquot C. Supportive treatment improves survival in multivisceral cholesterol crystal embolism. Am J Kidney Dis 1999;33:840-50.

\section{At what cost are resuscitation discussions avoided in general practice?}

Editor - Further to the assertion by Fritz et al (Clin Med December 2014, pp 571-6) that resuscitation, alongside overall goals of care, should be routinely discussed in clinical practice, the benefits of initiating discussions in the community warrant attention.

Deciding treatment priorities at the end of life requires space for deliberation. Particularly when acutely unwell or lacking capacity, it is not always possible for patients to engage with these discussions meaningfully in hospital. An equitable standard of care can only be achieved if end of life conversations and advance care planning are conducted routinely by GPs.

Most patients identified by GPs as nearing the end of life have not had resuscitation decisions documented; in 502 practices, 
only $20 \%$ of these patients had a community DNACPR form. ${ }^{1}$ Despite death being predicted, this can expose patients to unsuccessful paramedic resuscitation attempts in their final moments. Neither though should DNACPRs be misinterpreted to mean 'do not admit to hospital.' Reports suggesting paramedics feel they 'cannot clinically help' such patients, diverting ambulances elsewhere, are highly concerning. ${ }^{2}$ Contextualisation amid overall goals of care remains key; much can be learnt from the US experience of POLST. ${ }^{3}$

Are GPs reluctant to discuss end of life care? The national snapshot indicated that $58 \%$ of dying patients were offered this conversation, with $42 \%$ documenting their preferences. ${ }^{1}$ GPs report particular anxieties when mentioning resuscitation, often waiting until the patient's condition clearly deteriorates. ${ }^{4}$ This is problematic. Doctors are systematically overoptimistic when prognosticating in terminal illness, which may guide patients to request futile treatment options at the expense of optimal supportive care. Though challenging, resuscitation discussions are necessary in achieving a peaceful death.

Delegating additional, ostensibly bureaucratic, responsibilities to GPs is unlikely to be popular. Are such discussions a priority when practices already strain to deliver an escalating workload with limited resources? From crisis comes opportunity. Acute trusts are similarly struggling to manage their flow of frail elderly with increasingly complex needs. Upholding patients' wishes at the end of life, with shared understanding between healthcare services - the best standard of care - could still prevent unnecessary hospital admissions. This would alleviate health budget pressures, releasing funding for reinvestment in general practice.

NICK CORK

Medical student, University of Cambridge School of Clinical Medicine,

Cambridge, UK

RODGER CHARLTON

Professor of primary care education, University of Nottingham,

Nottingham, UK

\section{References}

1 Omega - the National Association for End of Life Care. End of life care in primary care. Shrewsbury: Omega, 2009. Available online at www.omega.uk.net/admin/uploads/file/National\%20snapshot $\% 20$ of $\% 20$ end $\% 20$ of $\% 20$ life $\% 20$ care $\% 20-\% 20$ key $\% 20$ findings.pdf [Accessed 11 January 2015].

2 BBC News. East of England Ambulance 999 downgrade period saw 57 patients die. 12 January 2015. Available online at www.bbc.co.uk/ news/uk-england-30782092 [Accessed 5 February 2015].

3 Fritz Z and Barclay S. Patients' resuscitation preferences in context: lessons from POLST. Resuscitation 2014;85:444-5.

4 Miller S and Dorman S. Resuscitation decisions for patients dying in the community: a qualitative interview study of general practitioner perspectives. Palliat Med 2014;28:1053-61. 